Ultrathin Solar Cell with Magnesium-Based Optical Switching for Window Applications

This is the accepted proof of the paper published in IEEE Journal of Photovoltaics.

https://doi.org/10.1109/JPHOTOV.2021.3110311

Cite as:

M. Götz-Köhler, H. Meddeb, K. Gehrke, M. Vehse and C. Agert, "Ultrathin Solar Cell With MagnesiumBased Optical Switching for Window Applications," in IEEE Journal of Photovoltaics, vol. 11, no. 6, pp. 1388-1394, Nov. 2021, doi: 10.1109/JPHOTOV.2021.3110311

The copyright of the Work is with The Institute of Electrical and Electronics Engineers (IEEE)

(C) IEEE 2021 
1

\title{
Ultrathin Solar Cell With Magnesium-Based Optical Switching for Window Applications
}

\author{
Maximilian Götz-Köhler ${ }^{\circledR}$, Hosni Meddeb ${ }^{\circledR}$, Kai Gehrke, Martin Vehse, and Carsten Agert
}

\begin{abstract}
Photovoltaic windows that can be switched between transparent and energy harvesting mode can be realized by using ultrathin solar absorbers embedded in an optical nanocavity. In the present work, we use a $5 \mathrm{~nm}$ thick amorphous germanium absorber integrated in a magnesium-based thin film optical cavity, which switches from an absorptive to a transparent state due to hydrogen absorption. We analyze the influence of the mirror layer thickness on the light absorption, photocurrent generation, and transmission as well as color neutrality of the device. The optical properties are studied by 1-D transfer-matrix method by changing Mg thickness between 0 and $100 \mathrm{~nm}$, then compared to the experimental results of fabricated devices. When the thickness of $\mathrm{Mg}$ increases, the switchable average transparency varies between $25 \%$ and $0 \%$, while the power conversion efficiency rises up to $2.3 \%$. The applicability of the device is tested by modeling the annual power generation in realistic scenarios. The influence of the cardinal orientation and the seasons on the switchable photovoltaic window implemented in a building facade with the abovementioned parameters is analyzed for different switching scenarios.
\end{abstract}

Index Terms-Magnesium optical switching, smart window, switchable solar cell, ultrathin solar cell.

\section{I} N MODERN buildings, huge glass facades often separate the interior from the exterior environment. They are supposed to supply thermal and visual comfort to the inner of the building. To improve this comfort, shading systems have to be implemented, which provide effective light and heat management [1]-[3]. Dynamic daylight control can be provided by smart windows, which attracted great interest in recent years [4]-[8]. These windows are able to reduce the amount of energy needed for cooling in the building [9]. The emergence of switchable solar cells added a new energy harvesting capability to these smart windows. While still being able to dynamically block sunlight from entering the interior of a building, these smart solar windows generate electricity at the same time. For the realization of a smart photovoltaic window, a technical solution enabling reversible switching from a transparent state to an absorbing photovoltaic state is required. Different approaches are

Manuscript received March 30, 2021; revised July 9, 2021; accepted August 31, 2021. (Corresponding author: Maximilian Götz-Köhler.)

The authors are with the German Aerospace Center (DLR) Institute of Networked Energy Systems, 26122 Oldenburg, Germany (e-mail: maximilian.goetz@dlr.de; hosni.meddeb@dlr.de; kai.gehrke@dlr.de; martin. vehse@dlr.de; carsten.agert@dlr.de).

Color versions of one or more figures in this article are available at https: //doi.org/10.1109/JPHOTOV.2021.3110311.

Digital Object Identifier 10.1109/JPHOTOV.2021.3110311 applied to realize switchable solar cells such as thermochromic perovskite layers [10], [11], liquid crystal diffuse reflectors [12], photochromic dye sensitized solar cells, [13] or gasochromic methods [14], [15]. Most of these devices use semitransparent solar cells as a basis and add the appropriate scheme to vary the light absorption dynamically. Achieving a suitable transparency for window applications requires the implementation of special absorber layers, which can be found in existing transparent photovoltaics [16].

In this work, we further study a switchable solar cell based on a hydrogenated amorphous germanium (a-Ge:H) absorber layer and a gasochromic switchable $\mathrm{Mg} / \mathrm{Ti} / \mathrm{Pd}$ back contact as introduced previously [14], [15], [17]. The PV window utilizes the change of refractive index from $\mathrm{Mg}$ to $\mathrm{MgH}_{2}$ due to hydrogen absorption. This transition from a metallic to a dielectric layer allows the device to become transparent and reduce the overall absorption. Charge carriers are generated in the a-Ge:H layer with a thickness of only $5 \mathrm{~nm}$. Due to the low thickness of the nano-absorber, its size-dependent bandgap is dominated by quantum confinement [18]. The transparency in the window mode of the device is achieved by the low single pass absorption through the thin absorber layer and the comparable high transparency of $\mathrm{MgH}_{2}$.

We focus on the impact of the $\mathrm{Mg}$ thickness on photocurrent generation, transparency, and color neutrality of the device. A point of highest change between absorbing and transparent state can be found separately for absorption as well as for transmission depending on the Mg thickness. Furthermore, the applicability of the device in a realistic scenario as a smart window including the power output and total transmitted sun light of the device is studied using a model for direct solar irradiation under clear sky conditions on switchable solar windows facing different cardinal directions in Oldenburg, Germany. The presented approach in this work can be generalized to PV windows in other geographical locations taking similar considerations and methods.

\section{EXPERIMENTAL DETAILS}

The solar cell with n-i-p configuration consists of hydrogenated amorphous/micro crystalline silicon and germanium multilayers, structured as (n-a-Si:H/i-a-Si:H/i-a-Ge:H/i- $\mu \mathrm{c}$ $\mathrm{Si}: \mathrm{H} / \mathrm{p}-\mu \mathrm{c}-\mathrm{Si}: \mathrm{H})$ with thicknesses $(7 \mathrm{~nm} / 5 \mathrm{~nm} / 5 \mathrm{~nm} / 5 \mathrm{~nm} / 7 \mathrm{~nm})$. We use soda lime glass substrates (size: $10 \times 10 \mathrm{~cm}^{2}$ ) coated with $1000 \mathrm{~nm}$-thick $\mathrm{ZnO}$ :Al as front contact. The back contact is based on $\mathrm{Mg}$ with different thicknesses to ensure the optical switching. A Pd capping layer $(5 \mathrm{~nm})$ is added to prevent 


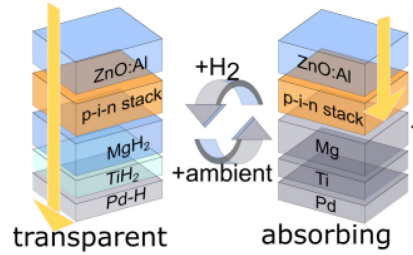

(a)

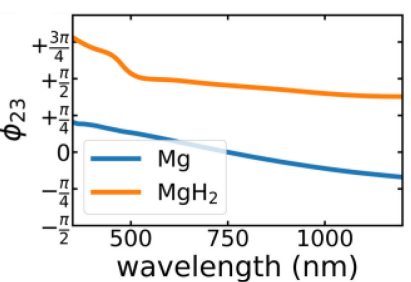

(b)

Fig. 1. (a) Layer stack in transparent and absorbing state and (b) spectral phase change on reflection at the absorber/back contact interface. The blue curve shows the phase shift for an a-Ge-Mg interface, while the orange curve describes the a- $\mathrm{Ge}-\mathrm{MgH}_{2}$ interface.

the layers from oxidation and acts as catalyst for hydrogen absorption [19]. Between the $\mathrm{Mg}$ and the Pd layer, a thin Ti layer $(1 \mathrm{~nm})$ is deposited to prevent the alloying of $\mathrm{Mg}$ and Pd [20], [21]. To improve the electrical contact between $\mathrm{Mg}$ with a low work function and p-doped $\mu \mathrm{c}-\mathrm{Si}$, a $5 \mathrm{~nm}$ thin layer of $\mathrm{MoO}_{\mathrm{x}}$ is used as charge selective contact [22]. The switching process is initiated by exposing the device to $5 \% \mathrm{H}_{2}$ in $\mathrm{N}_{2}$ at atmospheric pressure and requires less than $15 \mathrm{~min}$. The gas mixture is nonexplosive and nonflammable. The optical properties of $\mathrm{Mg}$ drastically change when exposed to $\mathrm{H}_{2}$ [23] It switches from a metallic to a dielectric transparent state by incorporating hydrogen atoms, turning into magnesium hydride $\left(\mathrm{MgH}_{2}\right)$ [24]-[26]. Pd and Ti, which are also included in the layer stack only change their optical properties slightly due to hydrogen absorption [23]. The hydrogen desorption process takes place at ambient air and requires another $15 \mathrm{~min}$. The layer stack is shown in Fig. 1(a). We model the light absorption and transmission of the solar cell for both states of the back contact: $\mathrm{MgH}_{2}$ and $\mathrm{Mg}$. Thereby, we study the influence of the thickness of the $\mathrm{Mg}$ layer on the optical parameters. This is done by 1-D transfer-matrix method in the software package CODE/Scout by W. Theiss. The optical models consist of refractive index data generated from transmission and reflection data of single layers with same or comparable thickness. Optical data for $\mathrm{MgH}_{2}$ and $\mathrm{TiH}_{2}$ are taken from Palm et al. [23]. The modeling results are compared to experimental outputs of the fabricated solar cells. The absorber and doped layers are deposited by plasma enhanced chemical vapor deposition in a deposition tool from Leybold, Germany. $\mathrm{Mg}, \mathrm{Pd}, \mathrm{MoO}_{\mathrm{x}}$, and Ti layers are deposited in one pump down process in an electron beam evaporation chamber at a pressure of $9 \cdot 10^{-6}$ mbar. The tool is from VTD, Germany. The detailed deposition parameters for all layers can be found in the supplementary information.

Current-voltage (I-V) curves are measured with a WACOM solar simulator under AM1.5 standard conditions with $1000 \mathrm{~W} / \mathrm{m}^{2}$ irradiation. For each $\mathrm{Mg}$ thickness 16 cells are produced with an area of $1 \times 1 \mathrm{~cm}^{2}$ each. From these 16 cells the I-V-diode characteristics are extracted by fitting a single diode model with the python package pvlib (v 0.8.1) to each measurement curve [27]. Average values together with standard deviation were extracted for short circuit current and series resistance. We also show the I-V curves of the best performing cell of each configuration. Optical data for reflection and transmission was measured with a Cary $5000 \mathrm{UV}$-Vis spectrophotometer from Agilent. We used a white integrating sphere to measure the complete hemispherical transmission and reflection. The modeled transmission spectra of the device with $\mathrm{MgH}_{2}$ back contact is converted into CIE color space with the python package color (v.0.3.16) to analyze the color appearance [28].

The model to calculate the direct solar irradiation on to the switchable window is implemented in Python 3.85. It uses the azimuth and altitude data of the sun for the year 2017 generated with the python package pysolar (v 0.6) [29]. Starting with sunrise, the position of the sun is evaluated in 500 equally spaced steps of time between sunrise and sunset for each single day. The clear-sky irradiation from the sun on a perpendicular area of $1 \mathrm{~m}^{2}$ is given by the software package. The angle $\alpha$ between sun and solar window is calculated by converting the sun's position to Cartesian coordinates with the center of the solar window as coordinate origin and using of following equation with the standard scalar product:

$$
\cos (\alpha)=\frac{\overrightarrow{\mathrm{v}}_{\text {Sun }} \cdot \overrightarrow{\mathrm{u}}_{\text {window }}}{\left|\overrightarrow{\mathrm{v}}_{\text {Sun }}\right|\left|\overrightarrow{\mathrm{u}}_{\text {window }}\right|} .
$$

Here, $\vec{v}_{\text {Sun }}$ indicates the position of the sun and $\vec{u}_{\text {window }}$ describes the surface normal of the solar cell. This term is multiplied with the direct normal irradiation to get the irradiation received by a vertical mounted solar window [30]. The multiplication with the solar cell efficiency results in the power generated from the solar cell. It does not include reduction of efficiency for large angles or low irradiation [31]. The location of the solar window for the current model is Oldenburg in Germany at $53.15^{\circ} \mathrm{N}$ and $8.17^{\circ} \mathrm{E}$.

\section{RESULTS AND DISCUSSION}

The main aspects to be considered for semitransparent and switchable photovoltaics are the light transmission and photoconversion efficiency in different switching scenarios [32]. We focus on the influence of the $\mathrm{Mg}$ back contact on these parameters, as well as the application case of switchable windows in a building facade. First, we show the results from 1-D transfer matrix simulations followed by measurements of real devices. The simulated and measured parameters are then used to model the annual power conversion and the transmitted light of a switchable photovoltaic window for a pre-defined switching scenario.

The switchable absorption is established using a simplified multilayers configuration as shown in Fig. 1(a). The optical nanocavity consists of an absorbing medium with high extinction coefficient (n-i-p stack) and deeply subwavelength dimension [33]-[35] positioned between a front transparent conductive electrode ( $\mathrm{ZnO}: \mathrm{Al})$ and a metallic reflective back contact $(\mathrm{Mg})$. The absorption enhancement is established by a compensation of the phase shifts due to reflection of light at the front $\left(\varphi_{12}\right)$ and back $\left(\varphi_{23}\right)$ with the propagation phase shift [17], [33]-[35]. The phase-shift due to reflection at a single interface between two media can be calculated from the Fresnel reflection coefficient 


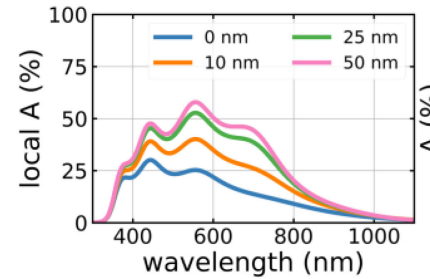

(a)

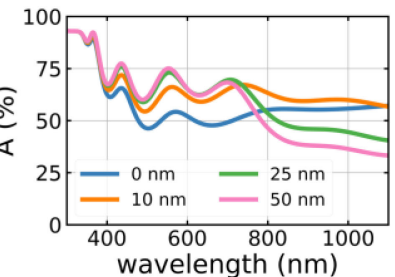

(b)
Fig. 2. (a) Simulation of switchable solar cell: Local absorption in the intrinsic $\mathrm{Si}$ and Ge layers and (b) total absorption of the complete device for different $\mathrm{Mg}$ thicknesses. The label " $0 \mathrm{~nm} \mathrm{Mg}$ " represents a cell stack without $\mathrm{Mg}$ layer, but with $1 \mathrm{~nm}$ Ti and $5 \mathrm{~nm} \mathrm{Pd}$.

$r_{i j}$ by its complex representation with the amplitude $r_{0}$ :

$$
r_{i j}=r_{0} \cdot e^{\mathbf{i} \phi_{i j}} .
$$

The phase shift $\varphi_{23}$ between n-i-p stack and $\mathrm{Mg}$ back mirror is drastically changed, when $\mathrm{Mg}$ turns into $\mathrm{MgH}_{2}$, as can be seen in Fig. 1(b) for an interface between a-Ge:H and bulk $\mathrm{Mg} / \mathrm{MgH}_{2}$. The phase-shift of the electromagnetic wave due to reflection between n-i-p stack and $\mathrm{MgH}_{2}$ is almost constantly equal to $\varphi_{23}$ $=\pi / 2$. It only increases for wavelengths below $500 \mathrm{~nm}$. The metallic $\mathrm{Mg}$ back contact leads to a different phase-shift. In the considered spectral range, $\varphi_{23}$ gradually decreases from $\varphi_{23}=$ $+\pi / 4$ to $\varphi_{23}=-\pi / 4$. This leads to a drastic change of the resonance inside the cavity. With metallic $\mathrm{Mg}$ as back contact, the enhanced absorption inside the layer stack is achieved. The absorption enhancement is switched "OFF," when the resonance conditions inside the cavity are disturbed. This is done when $\mathrm{Mg}$ is transformed into $\mathrm{MgH}_{2}$. The following results will show the consequences of this difference of the phase shift between $\mathrm{Mg}$ and $\mathrm{MgH}_{2}$.

In Fig. 2(b) the simulated combined absorption inside the intrinsic layers is plotted for four different thicknesses of metallic $\mathrm{Mg}$ as $A_{\text {local }}=A_{i-a-G e: H}+A_{i-a-S i: H}+A_{i-\mu c-S i: H}$. All cell stacks have $1 \mathrm{~nm}$ Ti and $5 \mathrm{~nm}$ Pd as topmost layers. The lines present solar cell stacks without $\mathrm{Mg}$ (only Ti and Pd rear contact) as well as 10,25 , and $50 \mathrm{~nm} \mathrm{Mg}$.The local absorption increases from no $\mathrm{Mg}(0 \mathrm{~nm})$ to 10 and $25 \mathrm{~nm}$. The difference becomes smaller between 25 and $50 \mathrm{~nm}$ back contact. Thinner Mg layers remain slightly transparent, reducing the light confined inside the absorber. Thicker $\mathrm{Mg}$ layers lead to an enhanced local absorption of light in the a-Ge:H photoactive layer. The overall absorption inside the absorbing layers of only $25 \mathrm{~nm}$ reaches a considerable level of $50 \%$ from the total incoming light. A similar trend can be seen for the total absorption of the complete device. Thicker back contact layers lead to an increased total absorption of light. Between a back-contact thickness of 25 to $50 \mathrm{~nm}$, the cavity effect is fully apparent and highest absorption is reached, with a maximum of $\mathrm{A}=80 \%$. The remaining $20 \%$ of light are lost due to reflection [14]. However, the difference in total and local absorption is the parasitic absorption in the doped layers and in the $\mathrm{ZnO}: \mathrm{Al}$ front contact.

Fig. 3(a) presents the simulated transmission spectra after hydrogen absorption of the switchable photovoltaic window for different thicknesses of $\mathrm{MgH}_{2}$. The transmission decreases

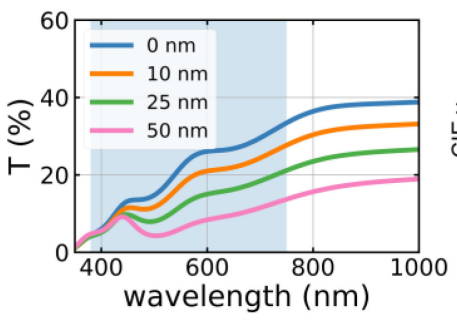

(a)

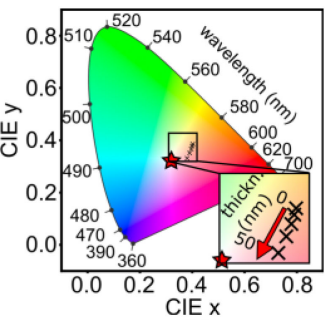

(b)
Fig. 3. (a) Simulated spectral transmission of complete device for four different $\mathrm{MgH}_{2}$ thicknesses, the blue shaded area represents the visible wavelength range. (b) CIE 1931 Chromaticity Diagram with $2^{\circ}$ Standard observer of transmitted color appearance. Black crosses in the center present the color range for varying thickness of $\mathrm{MgH}_{2}$ in solar cell "OFF" mode, the red star represents the white point in the CIE diagram.

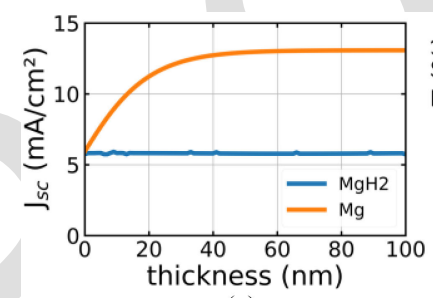

(a)

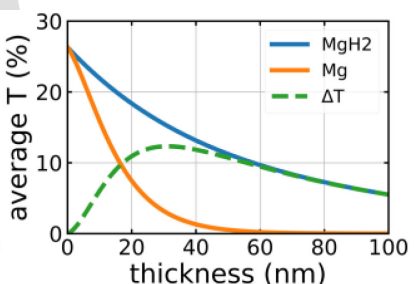

(b)
Fig. 4. (a) Evolution of photocurrent and (b) evolution of visible transmittance in dependence of the back-contact thickness. The blue curve shows the data for a solar cell with metallic $\mathrm{Mg}$, while the orange curve describes the more transparent cell with $\mathrm{MgH}_{2}$ back contact. The dashed green line indicates the difference in transmittance of both back contact variations.

gradually by increasing the thickness of the $\mathrm{MgH}_{2}$ layer. The transmission remains comparable for wavelengths below $\lambda=$ $450 \mathrm{~nm}$ due to the dominance of the n-i-p semiconductor layers in this spectral range and the refractive index change of $\mathrm{MgH}_{2}$ towards smaller wavelength [23]. $\mathrm{MgH}_{2}$ is not fully transparent, since it has an extinction coefficient $k>0$ over a wide spectral range. Therefore, more light is absorbed when its thickness increases. The transmission decreases from long to short wavelengths, since single pass absorption in the absorber, buffer and doped layers decrease the overall transparency. Fig. 3(b) presents the chromaticity diagram of the color appearance of the layer stack in CIE space for transmitted light. The graph shows the color appearance of the device for $\mathrm{MgH}_{2}$ thicknesses varied from 1 to $50 \mathrm{~nm}$. Overall, the device shows a high color neutrality. With increasing thickness, the color position in CIE space gets closer to the central white point (marked by the red star), but the overall transmission of the device decreases.

From the simulation of the local absorption in Fig. 2(a), the photocurrent can be calculated by the multiplication of the absorption spectra $A(\lambda)$ with the internal photo conversion efficiency $\eta(\lambda)$ and the photon flux of the AM1.5 solar spectrum $I_{\text {flux AM1.5 }}(\lambda)$ and their integration over the whole spectral range.

For the purpose of simplification, we assume an internal conversion efficiency of $\eta(\lambda)=1$. The result indicates the maximum achievable current from the corresponding nano-absorber. Fig. 4(a) presents the simulated photocurrent in "ON" and "OFF" state for different back contact thicknesses. With metallic $\mathrm{Mg}$ as 
back contact, the photocurrent from the intrinsic layers increases until it reaches a constant value of $J_{\mathrm{sc}}=13 \mathrm{~mA} / \mathrm{cm}^{2}$ beyond a Mg thickness of $43 \mathrm{~nm}$. The photocurrent generated in the transparent mode remains almost constant at a value of $J_{\mathrm{sc}}=$ $5.7 \mathrm{~mA} / \mathrm{cm}$. The difference between photocurrent in "ON" and "OFF" state reaches its highest value of $7.3 \mathrm{~mA} / \mathrm{cm}^{2}$ at $43 \mathrm{~nm}$ where $\mathrm{Mg}$ is almost completely opaque and no light can leave the cavity by transmission. This is also linked to the optimum reflection from the $\mathrm{Mg}$ rear mirror leading to higher absorption in the ultrathin solar cell.

Fig. 4(b) shows the average transmittance of the cell in both states calculated by following formula to cover a broad spectral range of the solar irradiation:

$$
T=\frac{\int_{350}^{1000} I_{\mathrm{AM} 1.5}(\lambda) \cdot T(\lambda) d \lambda}{\int_{350}^{1000} I_{\mathrm{AM} 1.5}(\lambda) \cdot d \lambda} .
$$

Hereby $T(\lambda)$ denotes the transmission spectrum obtained from the optical simulation. $I_{\mathrm{AM} 1.5}$ is the standard AM1.5G sun spectrum given in $\mathrm{Wm}^{-2} \mathrm{~nm}^{-1}$. On the one hand, the transparency of the cell stack in "ON" mode decreases drastically with increasing $\mathrm{Mg}$ thickness, owing to the metallic nature of the material. The visible transmittance becomes zero for thicknesses beyond $43 \mathrm{~nm}$. This corresponds to the point of saturation of the photocurrent in Fig. 4(a). The "OFF" state curve is also characterized by a decay of visible transmittance with increasing the back-layer thickness. The decay slope of the OFF-state is not as steep as the "ON" state. A cell with $25 \mathrm{~nm} \mathrm{MgH}_{2}$ has a transparency of $T_{\mathrm{vis}}=18 \%$. Thinner layers can reach values up to $T_{\mathrm{vis}}=25 \%$. For a thickness of $40 \mathrm{~nm}$, the cell would still show a $T_{\text {vis }}=14 \%$ in the transparent state. The difference in transmission between both states reaches a maximum for a thickness of $25 \mathrm{~nm}$. In this region, the optical difference between $\mathrm{Mg}$ and $\mathrm{MgH}_{2}$ has the highest impact on the optical switching of the solar cell. It is interesting to notice that the point of highest impact lies at different values for photocurrent and visible transmittance. This is related to the fact that the photocurrent depends on the condition of the optical field inside the cavity. If the $\mathrm{Mg}$ layer absorbs hydrogen, its transparency and reflectivity change simultaneously [36], [37]. This has a strong influence on light trapping inside the cavity as shown above. $\mathrm{Mg}$ layers with a thickness above $43 \mathrm{~nm}$ are almost completely opaque. Therefore, no further change in transmission can be measured for thicker layers, whereas, thinner layers of $\mathrm{Mg}$ are still partially transparent to visible light, which reduces the change of transparency, in case hydrogen is absorbed. Therefore, the thickness of the Mg layer influences the absorption as well as the transparency both in an opposite way when hydrogen is absorbed.

After the modeling study of the switchable layers stack, the fabrication and the optoelectronic characterization of solar cell devices are performed. Fig. 5(a) presents the measured J-V curves of five switchable solar cells with different thicknesses of the $\mathrm{Mg}$ back contact, comparable to the modeling conditions. For all investigated $\mathrm{Mg}$ thicknesses diode behavior of the cells can be seen. However, the J-V curves deviate from ideal behavior by the low fill factor (FF) level. The FF lays between 40 and 45\%,
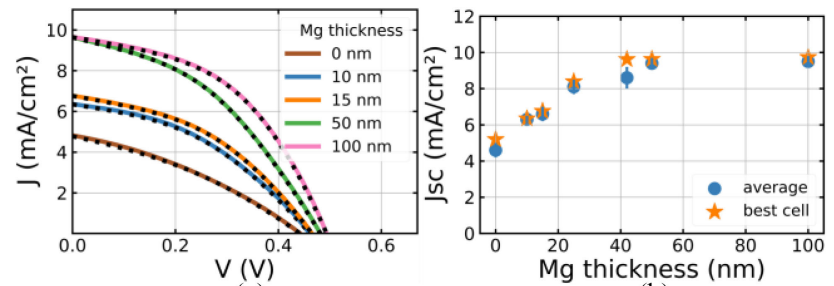

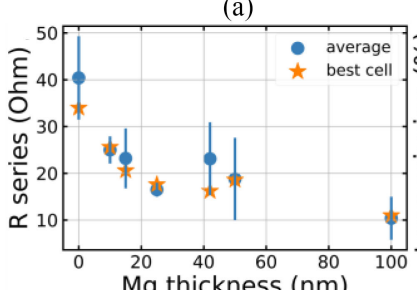

(c)

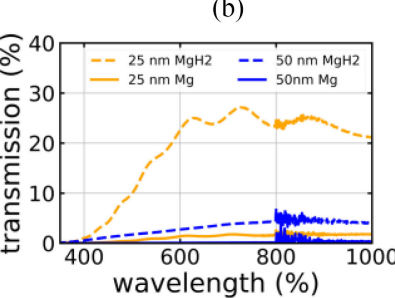

(d)
Fig. 5. (a) Measured JV curves of switchable solar cells with 0 to $100 \mathrm{~nm}$ $\mathrm{Mg}$ thickness. The dashed lines present single diode fits while the colored lines present the measured data. (b) Evolution of short circuit current with $\mathrm{Mg}$ thickness. (c) Evolution of series resistance from the single diode fits. (d) Transmission plots for solar cells with 25 and $50 \mathrm{~nm} \mathrm{Mg}$ back contact in absorptive (solid line) and transparent (dashed line) state.

due to the high series resistance induced by the thin Mg contact layers as well as the low parallel shunt resistance resulting from the impurities and recombination sites at the interface between absorber and electrical contact. The open circuit voltage $\left(V_{\mathrm{OC}}\right)$ and the FF slightly increase for thicker Mg layers. This can be explained by a higher shunt resistance due to lower leakage currents at the junction periphery with $\mathrm{Mg}$ [38], as well as more absorption of light. In our study, the short circuit current changes from $J_{\mathrm{SC}}\left(t_{\mathrm{Mg}}=0 \mathrm{~nm}\right)=4.5 \mathrm{~mA} / \mathrm{cm}^{2}$ to $J_{\mathrm{SC}}\left(t_{\mathrm{Mg}}=100 \mathrm{~nm}\right)=$ $9.8 \mathrm{~mA} / \mathrm{cm}^{2}$ with increasing $\mathrm{Mg}$ thickness. The evolution of $J_{\mathrm{sc}}$ in dependence on the Mg thickness is displayed in Fig. 5(b). As predicted by the model, the current increases drastically until a thickness of around 40 to $50 \mathrm{~nm}$ of $\mathrm{Mg}$ is reached. After this point, the photocurrent seems to stays constant independent of the $\mathrm{Mg}$ thickness. Even though, the photocurrent is less than expected from the simulation, the thicker $\mathrm{Mg}$ layer is able to enhance the absorption inside the cavity, and therefore, improves the photocurrent. Another important point, which was not considered in the optical simulation, is the dependence of the series resistance of the solar cell on the Mg layer thickness. The thickness of the Mg layer influences the series resistance, since the switchable mirror functions not only as back side of the optical cavity, but also as an electrical contact of the cell. Therefore, thicker layers of $\mathrm{Mg}$ are able to transport the current with less loss and decrease the overall series resistance. The average value of the series resistance decreases from 40 to $10 \Omega$ with $\mathrm{Mg}$ thickness from 0 to $100 \mathrm{~nm}$, as demonstrated in Fig. 5(c). This influences the FF of the diode curves significantly. It can be clearly seen [in Fig. 5(a)], that the slope of the curves close to open circuit condition increases significant for increasing $\mathrm{Mg}$ thickness. The overall power conversion efficiency (PCE) of the devices (not shown here) reflect this behavior: The solar cell without $\mathrm{Mg}$ rear contact reaches an efficiency of only $0.8 \%$ due to the low absorption enhancement, high series resistance and low FF, whereas, a solar cell with $50 \mathrm{~nm} \mathrm{Mg}$ achieves a 


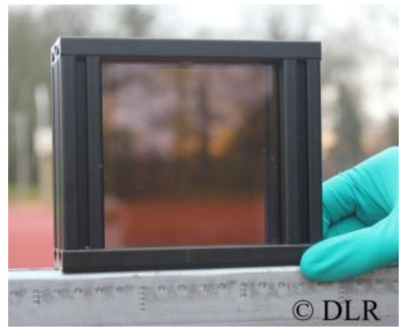

Fig. 6. Image of switchable photovoltaic window with $25 \mathrm{~nm} \mathrm{MgH}_{2}$ in transparent state.

photoconversion efficiency of about 2.0\%. For $100 \mathrm{~nm}$ Mg rear contact, the efficiency only increases slightly up to $2.3 \%$.

Fig. 5(d) shows the change of transparency of the cell with 25 and $50 \mathrm{~nm}$-thick $\mathrm{Mg}$ back contact, respectively. With $25 \mathrm{~nm}$ metallic $\mathrm{Mg}$ as back contact, the cell is almost completely opaque. The remaining transmission reduces the photocurrent generation of the device as discussed before. After absorption of hydrogen, the transmission of light is increased up to values of $30 \%$, which confirms the simulated results. The device with $50 \mathrm{~nm}$ thick $\mathrm{Mg}$ back contact is completely opaque in the absorptive state. After hydrogen absorption it becomes slightly transparent, but does not exceed 5\% transmission. This is significantly lower than predicted from the simulation and can be explained by the fact that $\mathrm{Mg}$ does not turn completely to $\mathrm{MgH}_{2}$ for layers exceeding a certain thickness level. Thus, the absorbed hydrogen could not penetrate through the complete depth of the layer. The formation of $\mathrm{MgH}_{2}$ close to the interface with Ti creates a diffusion barrier and $\mathrm{H}_{2}$ cannot penetrate deeper into the material [39], [40]. A possible solution to prevent this blocking layer formation would be the integration of additional Ti interface layers in the back contact, as demonstrated for thicker Mg layers [41].

Our experimental and simulation results demonstrate the feasibility of a switchable photovoltaic window based on a gasochromic $\mathrm{Mg} / \mathrm{Ti} / \mathrm{Pd}$ back reflector. The solar cell is able to reach significant absorption of light with only $5 \mathrm{~nm}$ a-Ge:H absorber layer. The moderate efficiency of $2.0 \%$ with switchable back contact might be sufficient to improve the ecological footprint of buildings with large glass façades by its dynamic shading capacity [9]. One important aspect is the excellent color neutrality of the transparent solar cell [see Fig. 3(b)]. This color neutrality can also be seen in the photograph in Fig. 6, showing a transparent solar cell with $25 \mathrm{~nm} \mathrm{MgH}_{2}$. The integration of the device into the building skin could be realized in a double-glazing configuration with the switchable solar cell embedded in the inner air-gap. When introducing the nonflammable switching gases through a gas inlet into the interspace, the cell can be put into "ON" or "OFF" state. To achieve high cyclability of the switchable solar cell, the interface between the switchable mirror layers and the absorber stack needs to be further adapted to preserve the underlying functional layers and prevent their deterioration induced by the volume expansion of the Mg-hydride compared to the bare $\mathrm{Mg}$ layer. Using metal alloys and interlayers between absorber and mirror might help reducing the detrimental effects. High cyclability of
4000 cycles of switching has been demonstrated for applications like switchable mirrors based on gasochromic Mg structures [42]. This shows that applying this technology to thin film solar cells might be a promising approach for long-time durable switchable photovoltaics.

Further improvements and considerations are required for the specific implementation of the previously demonstrated device technology in real buildings. Nevertheless, the estimation of the power generation capability and the indoor irradiation of such switchable photovoltaic windows is desired [43]. Therefore, we created a model that tracks the direct irradiation to a switchable window with $1.5 \%$ efficiency in "ON" mode and $18 \%$ transmission in "OFF" mode, installed vertically in the building façade. For this model, we only consider direct irradiation and neglect diffuse light, since we want to focus on the usage of the PV window as a sun blind. We consider windows facing in three different cardinal directions: south (S), south-east (SE), and west (W). Hereby, south describes a window on the northern hemisphere, facing in the direction of the equator. The windows are installed vertically in the building facade and are not adjusted like typical solar cells vertical to the radiation of the incident light. The position of the sun relative to the photovoltaic window determines the amount of direct irradiation by incident light on the surface. The maximum altitude of the sun, which is determined by the latitude of the location on earth, becomes a limiting factor for the generated power. High altitudes of the sun decrease the power generated from the PV window due to the large angle between sun and window normal [31]. The elevation and the azimuth of the sun also depend on the season. Power from direct irradiation can only be generated during times when the angle between sun and window is less than $90^{\circ}$. Irradiation profiles for vertical mounted PV have already been studied before [15] and are also well known from bifacial PV [30]. We focus here on solar energy conversion in the city of Oldenburg, Germany. The presented approach in this work can be generalized to PV windows in other geographical locations taking similar considerations and methods.

In order to analyze the performance of photovoltaic windows, the switching model scenarios are defined. There are several different approaches for the simulation of the switching behavior [9]. A threshold model can set a certain level of direct irradiation to trigger the switching process. Hereby, the window would be transparent while the irradiance is below the threshold value and switch into the photovoltaic state above this threshold. This model leads to the results displayed in Fig. 7. Here, the power output of a switchable photovoltaic window with $1.5 \%$ efficiency in "ON" and zero percent efficiency in "OFF" mode is shown. Fig. 7(a) presents the reference lines with the solar cell always switched "ON." While the $\mathrm{W}$ facing window shows a clear peak during June and July, the S and SE facing windows achieve the highest power conversion in March and October. This is a result of the higher direct irradiation onto these windows during the months of fall and spring. The converted power per day decreases, when the threshold switching is implemented. Fig. 7(b) shows the daily power outcome of the PV window for a switching threshold of $450 \mathrm{~W} / \mathrm{m}^{2}$ of direct irradiation. Due to the threshold, only at times of high direct irradiation the solar 


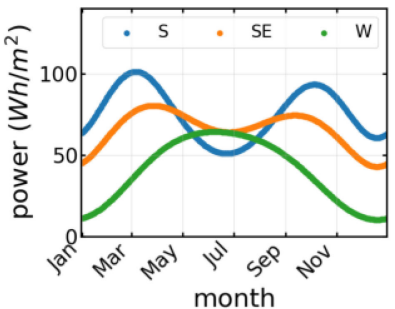

(a)

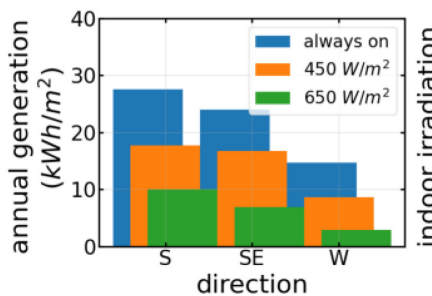

(c)

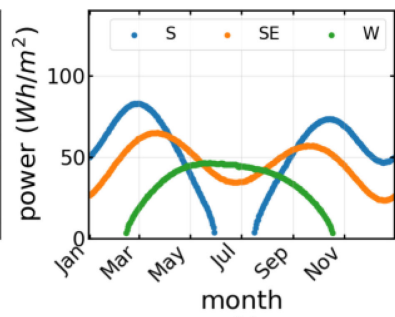

(b)

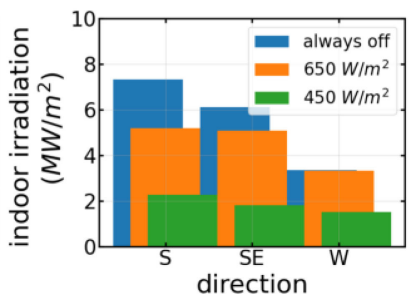

(d)

Fig. 7. (a) Generated power per day for $1.5 \%$ efficiency solar cell that is always "ON" or (b) only switched ON when the direct irradiation exceeds $450 \mathrm{~W} / \mathrm{m}^{2}$. (c) Annual power generation for three different switching scenarios. (d) Indoor irradiation for three different scenarios.

cell harvests energy for power conversion. There are several interesting consequences, which can be seen in Fig. 7(b). The W facing window still shows the highest power output during the summer months, where especially the irradiation during the afternoon onto the window is high. Nevertheless, this window does not generate any electricity from direct irradiation in the winter months. Since Oldenburg, Germany is located relatively far in the north, the sun sets too far in the south to have high irradiance onto this window in the winter. This would change for locations further south in the northern hemisphere, where the S-facing window would keep a high power-conversion from fall to spring, whereas, during the summer (June-August) the sun's elevation is too high and the angle between sun and window becomes unfavorable. The device remains in window mode and no energy is harvested.

Fig. 7(c) allows the comparison of annual power generation for three different thresholds of direct irradiation. The model shows that the power output of an $\mathrm{S}$ facing window would be reduced to $64 \%$ of the always on mode when the solar cell is only switched "OFF" above a threshold of $450 \mathrm{~W} / \mathrm{m}^{2}$. When the threshold is further increased to $650 \mathrm{~W} / \mathrm{m}^{2}$, the energy is still $36 \%$. The comparison between the cardinal direction shows that the $\mathrm{S}$ facing window always allows for the highest power outcome. The lack of direct irradiation during the summer months is compensated by high irradiation during fall, winter and spring season. For the $450-\mathrm{W} / \mathrm{m}^{2}$ threshold, only a slight reduction of power can be found between $\mathrm{S}$ and $\mathrm{SE}$ facing window from 17.7 to $16.7 \mathrm{kWh} / \mathrm{m}^{2}$ per year. The values shown here can contribute effectively in the reduction of the annual energy consumption of a building. Even though only clear sky irradiation is considered, the values give a guideline what to expect from switchable photovoltaic windows regarding their electricity generation in the façade. The indoor irradiation through the switchable solar cell with $18 \%$ average transmission is shown in Fig. 7(d). It decreases from $\mathrm{S}$ facing to $\mathrm{W}$ facing windows and from high to low switching value. It is interesting to see that a W facing window, which is always transparent and a window with switching threshold of $650{\mathrm{~W} \backslash \mathrm{m}^{2}}^{2}$ almost have the same annual indoor irradiation. This shows that almost no energy is absorbed any more for electricity generation with this setting. Considering the color neutrality of the device, the visual comfort of the interior remains, even though the overall irradiation is reduced. This also reduces the heat flux into the building improving the thermal comfort.

This model is a simplified approximation for the evaluation of the real illumination conditions throughout a complete year. Nevertheless, it shows that a combination of switchable photovoltaic windows facing in different directions could support the electricity generation during a year. Using the windows as solar cells to avoid dazzling under sunlight can replace conventional sun blinds and add a sun harvesting ability to windows. Higher electricity generation could be reached by increasing the efficiency of the device, but also by using it on tilted surfaces like rooftop windows for higher solar irradiation income.

\section{CONCLUSION}

This study reports on an ultrathin solar cell with a gasochromic-based optical switching enabled by Mg back contact. The thin Mg layer allows the device to switch the absorber, embedded in a nanocavity, between a transparent and an absorptive state, corresponding to "OFF" and "ON" modes of the optical cavity. While working as fully functional solar cell in the cavity "ON" state, the device turns into a transparent window mode after the absorption of hydrogen in the $\mathrm{Mg} / \mathrm{Pd}$ layers. The variation analysis of the $\mathrm{Mg}$ thickness using optical modeling allows the selection of a configuration with highest change in transmission and photocurrent generation. The experimental realization confirms a device with up to $2.3 \%$ efficiency and moderate transparency which can be switched "ON" and "OFF" while showing excellent color neutrality.

The presented device is a first step toward the realization of large scale switchable photovoltaic windows. The simulation of the power generation in different scenarios shows that by using our switchable solar cell technology, a power output of almost $20 \mathrm{kWh} / \mathrm{m}^{2}$ per year can be realized by using only direct sunlight. The accuracy of the presented simulations will be further improved by using real weather data and including also diffuse light. The successful demonstration of the promising potential for such switchable solar cells can pave the route for further valuable contribution to existing BIPV concepts.

\section{ACKNOWLEDGMENT}

The authors would like to thank N. Neugebohrn for providing code for data analysis in Python, U. Banik, C. Lattyak, and N. Osterthun for fruitful discussions and supporting this publication, and D. Berends for sputter deposition of AZO layers. 


\section{REFERENCES}

[1] S. D. Rezaei, S. Shannigrahi, and S. Ramakrishna, "A review of conventional, advanced, and smart glazing technologies and materials for improving indoor environment," Sol. Energy Mater. Sol. Cells, vol. 159, pp. 26-51, 2017.

[2] P. Lemarchand, J. Doran, and B. Norton, "Smart switchable technologies for glazing and photovoltaic applications," Energy Procedia, vol. 57, pp. 1878-1887, 2014.

[3] P. Lemarchand, E. McLean, and B. Norton, "Switchable windowsSpectral Transmission and switching times," in Proc. IEA SHC Int. Conf. Sol. Heating Cooling Buildings Ind., 2017, pp. 1-11.

[4] J. Li et al., "Transmittance tunable smart window based on magnetically responsive 1D nanochains," ACS Appl. Mater. Interfaces, vol. 12, no. 28, pp. 31637-31644, Jul. 2020.

[5] Y. Wang, E. L. Runnerstrom, and D. J. Milliron, "Switchable materials for smart windows," Annu. Rev. Chem. Biomol. Eng., vol. 7, pp. 283-304, Jun. 2016

[6] Y. Ke et al., "Smart windows: Electro-, thermo-, mechano-, photochromics, and beyond," Adv. Energy Mater, vol. 9, no. 39, 2019, Art. no. 1902066

[7] Y. Ke et al., "Emerging thermal-responsive materials and integrated techniques targeting the energy-efficient smart window application," $A d v$. Funct. Mater, vol. 28, pp. 1-18, 2018.

[8] C. G. Granqvist, "Electrochromics for smart windows: Oxide-based thin films and devices," Thin Solid Films, vol. 564, pp. 1-38, 2014.

[9] J.-M. Dussault and L. Gosselin, "Office buildings with electrochromic windows: A sensitivity analysis of design parameters on energy performance, and thermal and visual comfort," Energy Buildings, vol. 153, pp. 50-62, 2017.

[10] L. M. Wheeler et al., "Switchable photovoltaic windows enabled by reversible photothermal complex dissociation from methylammonium lead iodide," Nature Commun., vol. 8, no. 1, Nov. 2017, Art. no. 1722.

[11] J. Lin et al., "Thermochromic halide perovskite solar cells," Nature Mater., vol. 17, no. 3, pp. 261-267, Mar. 2018.

[12] J. Murray, D. K. Ma, and J. N. Munday, "Electrically controllable light trapping for self-powered switchable solar windows," ACS Photon., vol. 4 , no. 1, pp. 1-7, Jan. 2017.

[13] Q. Huaulmé et al., "Photochromic dye-sensitized solar cells with lightdriven adjustable optical transmission and power conversion efficiency," Nature Energy, vol. 5, no. 6, pp. 468-477, 2020.

[14] M. Götz et al., "Switchable photocurrent generation in an ultrathin resonant cavity solar cell," ACS Photon., vol. 7, no. 4, pp. 1022-1029, Apr. 2020.

[15] M. Götz, K. Gehrke, H. Meddeb, M. Vehse, and C. Agert, "Ultra-thin a-Ge:H solar cell with switchable absorption enhancement: Towards smart photovoltaic windows," in Proc. 47th IEEE Photovolt. Specialists Conf., Jan. 2021, pp. 1114-1118.

[16] C. J. Traverse, R. Pandey, M. C. Barr, and R. R. Lunt, "Emergence of highly transparent photovoltaics for distributed applications," Nature Energy, vol. 2, pp. 849-860, 2017.

[17] V. Steenhoff, M. Theuring, M. Vehse, K. von Maydell, and C. Agert, "Ultrathin resonant-cavity-enhanced solar cells with amorphous germanium absorbers," Adv. Opt. Mater, vol. 3, no. 2, pp. 182-186, Feb. 2015.

[18] H. Meddeb et al., "Quantum confinement-tunable solar cell based on ultrathin amorphous germanium," Nano Energy, vol. 76, 2020, Art. no. 105048.

[19] T. J. Richardson et al., "Switchable mirrors based on nickel-magnesium films," Appl. Phys. Lett., vol. 78, pp. 3047-3049, 2001.

[20] X. Duan, R. Griessen, R. J. Wijngaarden, S. Kamin, and N. Liu, "Selfrecording and manipulation of fast long-range hydrogen diffusion in quasifree magnesium," Phys. Rev. Mater., vol. 2, 2018, Art. no. 85802.
[21] J. Karst et al., "Optimizing magnesium thin films for optical switching applications: Rules and recipes," Opt. Mater. Exp., vol. 10, no. 6, 2020, Art. no. 1346.

[22] A. Alcañiz et al., "Germanium photovoltaic cells with MoOx holeselective contacts," Sol. Energy, vol. 181, pp. 357-360, 2019.

[23] K. J. Palm, J. B. Murray, T. C. Narayan, and J. N. Munday, "Dynamic optical properties of metal hydrides," ACS Photon., vol. 5, no. 11, pp. 4677-4686, Nov. 2018.

[24] R. Armitage, M. Rubin, T. Richardson, N. O'Brien, and Y. Chen, "Solidstate gadolinium-magnesium hydride optical switch," Appl. Phys. Lett., vol. 75 , no. 13, pp. 1863-1865, 1999.

[25] P. van der Sluis, "Optical switches of gadolinium-magnesium multilayers," Appl. Phys. Lett., vol. 73, no. 13, pp. 1826-1828, 1998.

[26] P. van der Sluis, M. Ouwerkerk, and P. A. Duine, "Optical switches based on magnesium lanthanide alloy hydrides," Appl. Phys. Lett., vol. 70, no. 25, pp. 3356-3358, 1997.

[27] W. F. Holmgren, C. W. Hansen, and M. A. Mikofski, "Pvlib python: A python package for modeling solar energy systems," J. Open Source Softw., vol. 3 , no. 29,2018 , Art. no. 884.

[28] T. Mansencal et al., "Colour 0.3.16," 2020, doi: 10.5281/zenodo.3757045.

[29] B. Stafford, "Pysolar documentation," 2020, Accessed: Jun. 2, 2020 [Online]. Available: https://pysolar.readthedocs.io/en/latest/\#

[30] S. Guo, T. M. Walsh, and M. Peters, "Vertically mounted bifacial photovoltaic modules: A global analysis," Energy, vol. 61, pp. 447-454, 2013.

[31] N. Martin and J. M. Ruiz, "Calculation of the PV modules angular losses under field conditions by means of an analytical model," Sol. Energy Mater. Sol. Cells, vol. 70, no. 1, pp. 25-38, 2001.

[32] A. A. F. Husain, W. Z. W. Hasan, S. Shafie, M. N. Hamidon, and S S. Pandey, "A review of transparent solar photovoltaic technologies," Renewable Sustain. Energy Rev., vol. 94, pp. 779-791, 2018.

[33] M. A. Kats, R. Blanchard, S. Ramanathan, and F. Capasso, "Thin-film interference in lossy, ultra-thin layers," Opt. Photon. News, no. 22, 2014 Art. no. 31545

[34] M. A. Kats and F. Capasso, "Optical absorbers based on strong interference in ultra-thin films," Laser Photon. Rev., vol. 10, no. 5, pp. 735-749, 2016.

[35] M. S. Unlu and S. Strite, "Resonant-cavity enhanced photonic devices," $J$. Appl. Phys., vol. 78, no. 2, pp. 607-639, Jul. 1995.

[36] Y. Yamada et al., "In situ spectroscopic ellipsometry study of the hydrogenation process of switchable mirrors based on magnesium-nickel alloy thin films," J. Appl. Phys., vol. 107, no. 4, Feb. 2010, Art. no. 043517.

[37] Y. Yamada, "Ellipsometric study of optical switching processes of Mg-Ni based switchable mirrors," Thin Solid Films, vol. 519, pp. 2941-2945, 2011, doi: 10.1016/j.tsf.2010.12.082.

[38] A. V. Shah and C. Droz, Thin-Film Silicon Solar Cells. Boca Raton, FL, USA: CRC Press, 2010.

[39] H. T. Uchida et al., "Absorption kinetics and hydride formation in magnesium films: Effect of driving force revisited," Acta Materialia, vol. 85, pp. 279-289, 2015, doi: 10.1016/j.actamat.2014.11.031.

[40] J. Rydén et al., "Unusual kinetics of hydride formation in Mg-Pd sandwiches, studied by hydrogen profiling and quartz crystal microbalance measurements," J. Less Common Metals, vol. 152, no. 2, pp. 295-309, 1989, doi: 10.1016/0022-5088(89)90097-0.

[41] H. Jung, S. Cho, and W. Lee, "A catalytic effect on hydrogen absorption kinetics in $\mathrm{Pd} / \mathrm{Ti} / \mathrm{Mg} / \mathrm{Ti}$ multilayer thin films," J. Alloys Compounds, vol. 635, pp. 203-206, Jun. 2015, doi: 10.1016/j.jallcom.2015.02.080.

[42] K. Tajima, Y. Yamada, S. Bao, M. Okada, and K. Yoshimura, "Flexible all-solid-state switchable mirror on plastic sheet," Appl. Phys. Lett., vol. 92, pp. 1-4, 2008, doi: 10.1063/1.2839298.

[43] K. Lee et al., "The development of transparent photovoltaics," Cell Rep. Phys. Sci., vol. 1, 2020, Art. no. 100143, doi: 10.1016/j.xcrp.2020.100143. 